\title{
The counterpart/s of IGR J20159+3713/SWIFT J2015.9+3715: dissecting a complex region with emission from keV to $\mathrm{TeV}$
}

\author{
L. Bassani ${ }^{1}$, R. Landi ${ }^{1}$, A. Malizia ${ }^{1}$, J. B. Stephen ${ }^{1}$, A. Bazzano ${ }^{2}$, A. J. Bird ${ }^{3}$, and P. Ubertini ${ }^{2}$ \\ 1 INAF/IASF Bologna, via Piero Gobetti 101, 40129 Bologna, Italy \\ e-mail: bassani@iasfbo.inaf.it \\ 2 INAF/IAPS Rome, via Fosso del Cavaliere 100, 00133 Rome, Italy \\ 3 School of Physics and Astronomy, University of Southampton, Highfield, SO17 1BJ, UK
}

Received 16 July 2013 / Accepted 15 October 2013

\begin{abstract}
We report on the identification of a new soft gamma-ray source, namely IGR J20159+3713/SWIFT J2015.9+3715, first detected by INTEGRAL/IBIS and then confirmed by Swift/BAT. The source, which has an observed $20-100 \mathrm{keV}$ flux in the range $(0.7-1.4) \times$ $10^{-11} \mathrm{erg} \mathrm{cm}^{-2} \mathrm{~s}^{-1}$, encloses a Fermi variable source (2FGL J2015.6+3709) and is spatially close to a TeV emitter (VER J2016+372). Thanks to X-ray follow-up observations performed with the X-ray telescope on board Swift, we have been able to identify the new IBIS/BAT detection with the combined emission of the blazar B2013+370 and the cataclysmic variable RX J2015.6+3711. Both objects show variability in X-rays, with the cataclysmic variable being the most variable of the two. At high energies (above $20 \mathrm{keV}$ ) the emission is likely dominated by B2013+370, but the contribution from RX J2015.6+3711 is not negligible, The blazar emits up to $\mathrm{GeV}$ frequencies where it is seen by Fermi, while the cataclysmic variable has a bremsstrahlung temperature which is too low to provide any contribution at these high energies. These findings also indicate that the INTEGRAL/Swift source is not associated with the $\mathrm{TeV}$ emission, which is most likely due to the supernova remnant (SNR)/pulsar wind nebula (PWN) CTB 87.
\end{abstract}

Key words. gamma rays: general - X-rays: general

\section{Introduction}

IGR J20159+3713/SWIFT J2015.9+3715 is a new soft gammaray source first detected with the IBIS imager on board the INTEGRAL satellite (Krivonos et al. 2010) and then confirmed by the BAT instrument on board the Swift mission (Cusumano et al. 2010). The source lies in a region of complex gamma-ray emission. In fact, IGR J20159+3713/SWIFT J2015.9+3715 is close to a GeV/Fermi/EGRET source (3EG J2016+3657/1FGL J2015.7+3708/2FGL J2015.6+3709) associated with the blazar B2013+370 (Halpern et al. 2001); the GeV emission is variable supporting the hypothesis of an extragalactic origin for the Fermi detection (Kara et al. 2012). Close by there is also a newly discovered TeV/VERITAS object (VER J2016+372), whose position is compatible with that of CTB 87 a supernova remnant $(\mathrm{SNR}) /$ pulsar wind nebula $(\mathrm{PWN})$ located at $6 \mathrm{kpc}$ in the Perseus arm, but in the line of sight of Cyg OB1 (Aliu et al. 2011). The properties and energetics of the underlying pulsar are poorly known although, recently, some information have been obtained by means of Chandra observations (Matheson et al. 2013). The association between the VERITAS and Fermi source seems to be excluded at the $99 \%$ confidence level (c.l.) as reported by Aliu et al. (2011), while the connection of these GeV and $\mathrm{TeV}$ sources with the IBIS/BAT detection has never been discussed before in the literature. To complicate the situation, we also note that IGR J20159+3713/SWIFT J2015.9+3715 has been identified with different source typology in the various hard $\mathrm{X}$-ray catalogues in which it is reported: as a SNR by Krivonos et al. (2010, 2012), as a cataclysmic variable (CV) in Cusumano et al. (2010), and as a blazar in the latest BAT 70-month survey (Baumgartner et al. 2013). This clearly reflects the complexity of this sky region and points to a reanalysis of all the available observations to understand the nature of the IBIS/BAT source and its relation to the nearby $\mathrm{GeV} / \mathrm{TeV}$ emitters.

Through a full analysis of these observations, we will demonstrate that the IBIS/BAT emission is likely due to the contribution of both the $\mathrm{CV}$ and the blazar and is unrelated to the SNR/PWN.

\section{Gamma-ray emission in and around IGR J20159+3713/SWIFT J2015.9+3715}

Figure 1 is a cut-off image of the region surrounding IGR J20159+3713/SWIFT J2015.9+3715 as seen by INTEGRAL/ IBIS in the $17-60 \mathrm{keV}$ band $^{1}$. It shows the 9-year average IBIS significance map with superimposed the source positional uncertainty (Krivonos et al. 2012); for comparison we also show the error circle reported in the latest BAT 70 month catalogue (Baumgartner et al. 2013). The source is detected by IBIS with a significance of $\sim 12 \sigma$ at a position corresponding to RA (J2000) $=20^{\mathrm{h}} 15^{\mathrm{m}} 31^{\mathrm{s}} .44$ and $\operatorname{Dec}(\mathrm{J} 2000)=+37^{\circ} 11^{\prime} 16^{\prime \prime} .8$, with an associated uncertainty of $\sim 3.4$ (90\% c.l.). The BAT detection is at $8 \sigma$ c.l. and its positional uncertainty is slightly larger but fully compatible with the IBIS one. The location of IGR J20159+3713/SWIFT J2015.9+3715 is 1.20 degrees above the Galactic plane, making difficult to discriminate whether it is a Galactic or an extragalactic object. A simple power law provides a good fit to the high-energy data and a photon index $\Gamma \sim 2 \mathrm{com}-$ bined to an observed $20-100 \mathrm{keV}$ flux in the range $(0.7-1.4) \times$ $10^{-11} \mathrm{erg} \mathrm{cm}^{-2} \mathrm{~s}^{-1}$ (Krivonos et al. 2012; Cusumano et al. 2010; Baumgartner et al. 2013).

\footnotetext{
1 Available at:

http://hea.iki.rssi.ru/integral/

nine-years-galactic-survey/index.php
} 


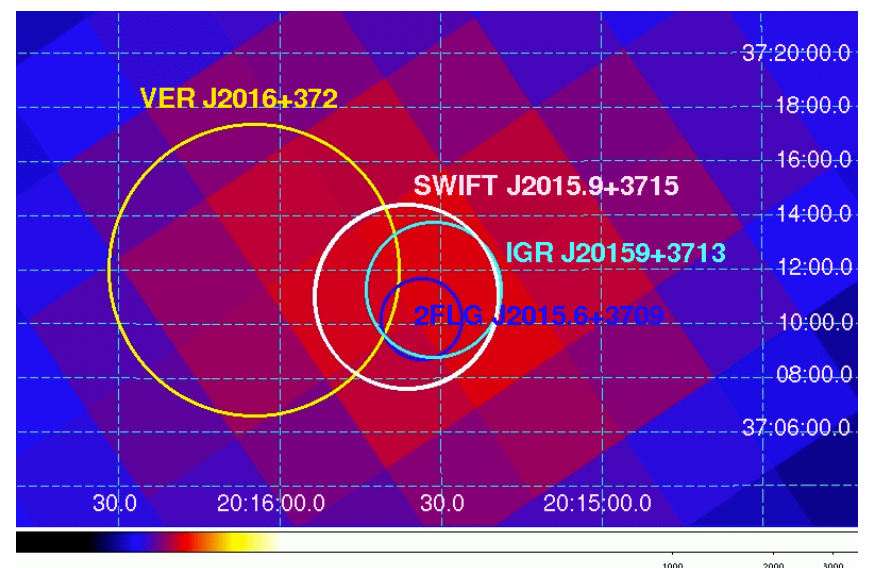

Fig. 1. IBIS 17-60 keV significance map containing IGR J20159+ $3713 /$ SWIFT J2015.9+3715. The white circle corresponds to the IBIS positional uncertainty, while the light blue is that from BAT 70 month survey; the small dark blue circle shows the positional uncertainty of the Fermi source, while the large yellow circle represents the area where the VERITAS emission comes from.

Here and in the following, spectral analysis is performed with XSPEC v.12.8.0 package and errors are quoted at $90 \%$ c.l. for one interesting parameter $\left(\Delta \chi^{2}=2.71\right)$.

As mentioned in the introduction, this hard X-ray source has different optical counterparts in the different catalogues in which it is listed, suggesting that either some associations are wrong or that the emission is from multiple objects.

The $\mathrm{GeV} / \mathrm{TeV}$ detections reported recently in the literature are also plotted for comparison in Fig. 1. For Fermi we used the position and relative uncertainty quoted by Kara et al. (2012) and based on 31 months of Fermi data; for the VERITAS detection we used the position reported by Aliu et al. (2011), taking as error the integration radius (0.09 degrees) employed to search for the TeV signal. As evident in Fig. 1, spatial considerations indicate a possible association between emissions in different wavebands, especially between hard X-ray and GeV/gamma-ray energies; it also emphasises the inability of current high-energy instruments to resolve a complex region such as this one.

The time-averaged Fermi spectrum is well described by a power law with photon index $\Gamma=(2.57 \pm 0.02)$ and a flux above $1 \mathrm{GeV}$ of $9.3 \times 10^{-9} \mathrm{~cm}^{-2} \mathrm{~s}^{-1}$; the Fermi spectrum is slightly softer than the one found previously by EGRET $(\Gamma=$ $(2.09 \pm 0.11)$, Hartman et al. 1999). Analysis of the Fermi light curve indicates flux variability and the presence of two flares in 2009 and 2010 (Kara et al. 2012). The presence of variability in the $\mathrm{GeV}$ emission, a property which is typical of blazars, clearly points to an association of the Fermi source with B2013+370, a flat-spectrum radio source which is still optically unclassified. On the basis of the source overall properties, Kara et al. (2012) concluded that B2013+370 is most likely a low-peaked BL Lac or a flat-spectrum radio quasar (FSRQ).

Above $1 \mathrm{TeV}$, the emission from VER J2016+372 is $\sim 0.8-1 \%$ that of the Crab Nebula and the spectrum is best fitted with a power law having a photon index $\Gamma=(2.1 \pm 0.5)$; the measured spectrum and the absence of variability observed throughout the $\mathrm{TeV}$ observation are properties similar to those of PWNs previously detected at TeV energies (Aliu et al. 2011). For this reason, the source has been associated with CTB 87, a SNR/PWN complex located at the centre of the TeV error circle, while its relation to B2013+370 has been considered unlikely.

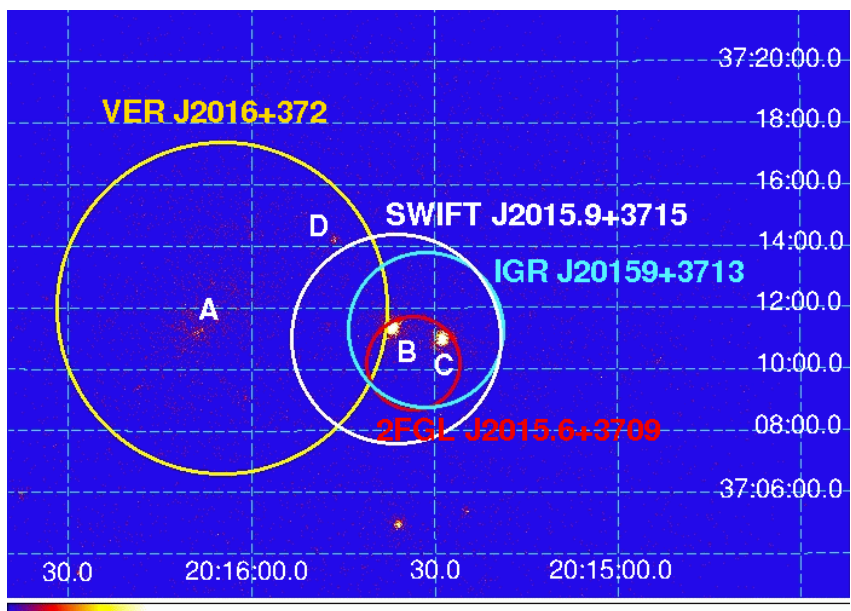

Fig. 2. XRT $0.3-10 \mathrm{keV}$ count map of the region surrounding IGR J20159+3713/SWIFT J2015.9+3715; error circles are the same as in Fig. 1.

\section{Swift/XRT follow-up observations}

\subsection{Imaging analysis}

In order to disentangle the emission from various sources and in different wavebands, a useful tool is to analyse the region at softer X-ray energies, where the instrument angular resolution and point source location accuracy are generally far better than at higher energies. To this purpose we used the Swift/XRT instrument, which has an angular resolution of 18 arcsec and a source location accuracy of a few arcsec (Burrows et al. 2005). Specifically, we used observations performed during the periods November 2006 and August 2010: eight pointings of reasonable exposure were carried out during these periods for a total time of $\sim 30 \mathrm{ks}$. XRT data reduction was performed using the XRTDAS standard data pipeline package (XRTPIPELINE v. 0.12.6), in order to produce screened event files. All data were extracted only in the photon counting (PC) mode (Hill et al. 2004), adopting the standard grade filtering (0-12 for PC) according to the XRT nomenclature.

Data from all eight observations were combined together in a single $0.3-10 \mathrm{keV}$ count map which is displayed in Fig. 2, together with all relevant error circles. The map clearly shows the presence of four X-ray sources, three brighter ones (A, B, and C) and one dimmer (D). Table 1 lists for each of these detections, the XRT position and uncertainty, the source extension, the detection significance in the $0.3-10 \mathrm{keV}$ energy band and above $4 \mathrm{keV}$, the source association and its nature.

The first source (A) is extended and the position of its brightest spot is compatible with the peak of the X-ray emission associated with the PWN recently discovered in CTB 87 (Safi-Harb 2012; Matheson et al. 2013). The source morphology is similar, although less detailed, to what is evident in a Chandra image of the region where both a compact nebula (with a torus/jet-like structure) and a diffuse nebula (with a cometary-like morphology) are clearly visible (Safi-Harb 2012; Matheson et al. 2013).

The second object (B) is associated with RX J2015.6+3711, one of the 14 bright ROSAT sources found within 3EG J2016+3657 error box and discussed in detail by Halpern et al. (2001). In their paper, RX J2015.6+3711 is source N.2 and its optical spectrum (display for the first time in Fig. 5 of Hapern et al. 2001) is that of a cataclysmic variable, probably of the magnetic type since its He II j4686 emission line is as strong 
Table 1. Swift/XRT detections.

\begin{tabular}{|c|c|c|c|c|c|c|c|c|}
\hline \multirow[t]{2}{*}{ Source } & \multicolumn{3}{|c|}{ Position } & \multirow[t]{2}{*}{ Extension } & \multicolumn{2}{|l|}{$\sigma$} & \multirow[t]{2}{*}{ Association } & \multirow[t]{2}{*}{ Type } \\
\hline & RA (J2000) & $\operatorname{Dec}(\mathrm{J} 2000)$ & $\begin{array}{c}\text { error } \\
(\operatorname{arcsec})\end{array}$ & & $(0.3-10 \mathrm{keV})$ & $(>4 \mathrm{keV})$ & & \\
\hline $\mathrm{A}^{a}$ & $20^{\mathrm{h}} 16^{\mathrm{m}} 08^{\mathrm{s}} .70$ & $+37^{\circ} 11^{\prime} 14^{\prime \prime} .10$ & $6 . " 00$ & extended & 17 & 7.9 & СТВ 87 & SNR/PWN \\
\hline $\mathrm{B}$ & $20^{\mathrm{h}} 15^{\mathrm{m}} 36^{\mathrm{s}} .95$ & $+37^{\circ} 11^{\prime} 21^{\prime \prime} .47$ & 3." 56 & point-like & 37 & 22 & RX J2015.6+3711 & $\mathrm{CV}$ \\
\hline $\mathrm{C}$ & $20^{\mathrm{h}} 15^{\mathrm{m}} 28^{\mathrm{s}} .76$ & $+37^{\circ} 10^{\prime} 58^{\prime \prime} .87$ & 3." 57 & point-like & 36 & 19.5 & B2 $2013+370$ & Blazar \\
\hline $\mathrm{D}$ & $20^{\mathrm{h}} 15^{\mathrm{m}} 46^{\mathrm{s}} .20$ & $+37^{\circ} 14^{\prime} 13^{\prime \prime} .20$ & $6 . " 00$ & point-like & 8 & 2.5 & X201546.42+371413.4 & unid \\
\hline
\end{tabular}

Notes. ${ }^{(a)}$ The position reported here coincides, within uncertainties, with that of the bright spot detected by Chandra within the SNR/PWN diffuse emission and is associated with the X-ray source CXOU J201609.2+371110, firstly detected by Matheson et al. (2013).

Table 2. Log of the Swift/XRT observations and count rates of each detection.

\begin{tabular}{lccccc}
\hline \hline Observation & Date & Exposure $^{a}$ & \multicolumn{3}{c}{$\begin{array}{c}\text { Count rate } \\
\text { blazar }\end{array}$} \\
& & $(\mathrm{s})$ & $\left(10^{-3}\right.$ counts s$\left.^{-1}\right)$ & $\left(10^{-3}\right.$ counts s $\left.^{-1}\right)$ & $\begin{array}{c}\text { PWN }^{c} \\
\left(10^{-3} \text { counts s}^{-1}\right)\end{array}$ \\
\hline$\# 1$ & July 12 2006 & 1528 & $40.1 \pm 5.2$ & $11.3 \pm 2.9$ & $1.3 \pm 0.9$ \\
$\# 2$ & Nov. 12 2006 & 4490 & $46.7 \pm 3.3$ & $22.6 \pm 2.3$ & $1.4 \pm 0.7$ \\
$\# 3$ & Nov. 17 2006 & 7367 & $66.8 \pm 3.0$ & $24.7 \pm 1.9$ & $13.9 \pm 1.4$ \\
$\# 4$ & Nov. 24 2006 & 170 & $87.6 \pm 24.5$ & $27.8 \pm 13.3$ & $10.6 \pm 8.4$ \\
$\# 5$ & Aug. 5 2010 & 962 & $57.9 \pm 8.0$ & $69.3 \pm 8.7$ & $7.9 \pm 3.3$ \\
$\# 5$ & Aug. 6 2010 & 7307 & $43.5 \pm 2.5$ & $55.6 \pm 2.8$ & $9.1 \pm 1.2$ \\
$\# 6$ & Aug. 22 2010 & 4310 & $43.3 \pm 3.2$ & $73.3 \pm 4.2$ & $8.8 \pm 1.5$ \\
$\# 8$ & Aug. 30 2010 & 4146 & $32.4 \pm 2.9$ & $46.4 \pm 3.4$ & $6.8 \pm 1.4$ \\
\hline \multicolumn{7}{c}{ Total } & 30280 & $48.4 \pm 1.3$ & $42.6 \pm 1.2$ & $10.2 \pm 0.6$ \\
\hline
\end{tabular}

Notes. ${ }^{(a)}$ Total on-source exposure time; ${ }^{(b)}$ the count rate is estimated in the $0.3-10 \mathrm{keV}$ energy range; ${ }^{(c)}$ the PWN count rate has been extracted from a circle of $20^{\prime \prime}$ radius centred on the brightest X-ray spot.

as $\mathrm{Hb} \mathrm{j4861;} \mathrm{the} \mathrm{spectrum} \mathrm{is} \mathrm{also} \mathrm{reddened,} \mathrm{implying} \mathrm{a} \mathrm{source}$ distance greater than $1 \mathrm{kpc}$.

The third source $(\mathrm{C})$ is coincident with the blazar B2013+370. Note that the distance between the CV and the blazar is only 1.6 arcmin, thus making the two unresolved at high energies.

Finally, the last much dimmer object coincides with a Chandra source, but has no clear association at other wavelengths (Ptak \& Griffiths 2003).

A few notes can be made on the basis of Fig. 2: IBIS error circle excludes the SNR/PWN CTB 87, thus implying a wrong association in the Krivonos et al. $(2010,2012)$ catalogues. Fermi error circle is interestingly compatible with both BAT 70-month and IBIS error circles, suggesting some association and highlighting the possibility that beside the blazar also the $\mathrm{CV}$ could be considered a less likely but still a possible $\mathrm{GeV}$ emitter. Finally, within the VERITAS positional uncertainty the SNR/PWN remains the most likely candidate, although source D cannot be totally disregarded.

Imaging this region of the sky above $4 \mathrm{keV}$ could be of some help: as shown in Table 1, the CV and the blazar are still the brightest objects detected at these higher energies (at 22 and $19.5 \sigma$, respectively), the SNR/PWN is still seen but only at $7.9 \sigma$, while the fourth object dissapears below the $2.5 \sigma$ detection level. While this evidence does not allow a clear identification of the Fermi and VERITAS counterparts, it certainly helps to clarify which is the real association to the IGR J20159+3713/SWIFT J2015.9+3715 source. Simply on the basis of spatial and brightness considerations, both the $\mathrm{CV}$ and the QSO are the most likely counterparts of the hard X-ray detection. Further clues may come from spectroscopic considerations as well as variability studies.

\subsection{Spectral analysis}

Events for spectral analysis were extracted within a circular region of radius $20^{\prime \prime}$ (which encloses about $90 \%$ of the PSF at $1.5 \mathrm{keV}$, Moretti et al. 2004) centred on the position of each interesting source. The background was extracted from various source-free regions close to the X-ray source using both circular/annular regions with different radii, in order to ensure an evenly sampled background. In all cases, the spectra were extracted from the corresponding event files using XSELECT software and binned using GRPPHA in an appropriate way, so that the $\chi^{2}$ statistic could be applied. We used the latest version (v.013) of the response matrices and create individual ancillary response files (ARF) using XRTMKARF v.0.6.0. In all our fitting procedures we have used a Galactic column density which in the direction of these sources is $1.15 \times 10^{22} \mathrm{~cm}^{-2}$ (Kalberla et al. 2005).

Table 2 reports for each X-ray measurement, the observation date, the relative exposure, the net count rate in the 0.3-10 keV energy band and the detection significance for the CV RX J2015.6+3711, the blazar B2013+370 and the SNR/PWN CTB 87. There is clear evidence that all sources underwent some flux changes over the XRT monitoring: the reduced $\chi^{2}$ (for a constant model at the mean) for sources A, B and $\mathrm{C}$ is $15,50,20$, respectively, which indicates that all three objects changed flux in time with the $\mathrm{CV}$ being the most variable of the three. Variability in blazars, CV and even in PWN is not unexpected and can be used to characterise further these objects.

Unfortunately, it also complicates the picture, expecially in the case of B2013+370 and RX J2015.6+3711, where it becomes quite difficult to disintangle the contribution of one source from the other at high energies purely on the basis of flux variations. Spectral information can be useful, in this respect and therefore, to enhance the signal to noise ratio and obtain good constrain on the source parameters, we have combined XRT data 


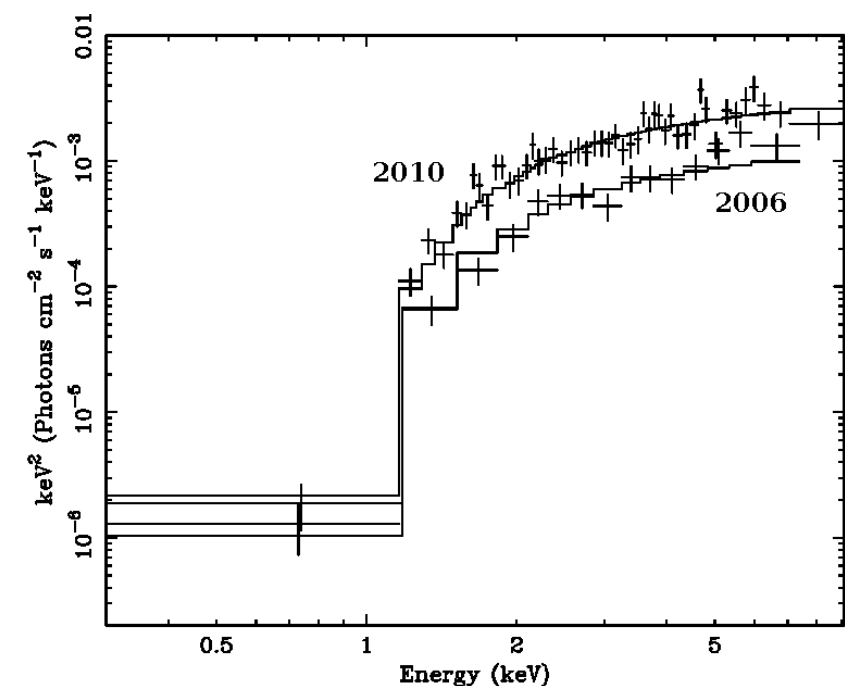

Fig. 3. Two-epoch XRT spectra of the blazar B2013+370 fitted simultaneously with an absorbed power law.

from two-epoch observations (2006 and 2010) to obtain two states of the blazar flux, while all CV data have been added together to study the source average X-ray spectrum.

The two blazar spectra have been fitted with the same model, i.e. an absorbed power law with different $1 \mathrm{keV}$ normalisations; this is justified by the fact that within uncertainties Kara et al. (2012) report a similar XRT spectum for the 2006 and 2010 periods. The absorption is partially Galactic and partially intrinsic to the source. The best fit $\left(\chi^{2} / v=66.4 / 59\right)$ is obtained with a photon index of $\Gamma=\left(1.66_{-0.20}^{+0.22}\right)$ and an intrisic column density of $\left(0.78_{-0.29}^{+0.33}\right) \times 10^{22} \mathrm{~cm}^{-2}$; the $2-10 \mathrm{keV}$ flux is $\sim 2 \times 10^{-12} \mathrm{erg} \mathrm{cm}^{-2} \mathrm{~s}^{-1}$ and $\sim 5 \times 10^{-12} \mathrm{erg} \mathrm{cm}^{-2} \mathrm{~s}^{-1}$ for the 2006 and 2010 period, respectively (see Fig. 3$)^{2}$. The extrapolation of these two spectra to high energies provides a $20-100 \mathrm{keV}$ flux in the range $(0.5-1.2) \times 10^{-11} \mathrm{erg} \mathrm{cm}^{-2} \mathrm{~s}^{-1}$.

Note that the addition of an intrinsic column density provides a significant improvement in the fit (>99.99\% c.l. using the F-test). Intrinsic absorption is a common property of radioloud/high-redshift blazars (Page et al. 2005) and it is generally ascribed to the presence of absorbing material in the jet. An alternative possibility is that the continuum is intrinsically curved (Tavecchio et al. 2007) as expected if the emission is described by a broken power law: however, this model does not fit the data properly leaving absorption as the only viable scenario. Overall, we can conclude that the XRT spectra of B2013+370 indicate different source fluxes over time and also suggest that this object can provide a large fraction of the flux seen above $20 \mathrm{keV}$.

The soft X-ray spectrum of the CV is more difficult to characterise. As reported by Landi et al. (2009), magnetic CVs are generally described by various spectral components, more specifically a black body model plus an absorbed bremsstrahlung one, both passing through the Galactic column density. The absorption related to the bremsstrahlung component covers the source only partially (pcfabs model in XSPEC). Such model, if applied to the average XRT spectrum of RX J2015.6+3711, is feasible, but provides a bremsstrahlung temperature which is too high ( $k T \geq 70 \mathrm{keV}$ ). Ideally, one needs to have broadband coverage for such a detailed analysis, but this is difficult for RX J2015.6+3711 given the significant contamination from

\footnotetext{
2 Note that in Kara et al. (2012) the 2006 flux is brighter than the 2010 one, possibly due to a misprint.
}

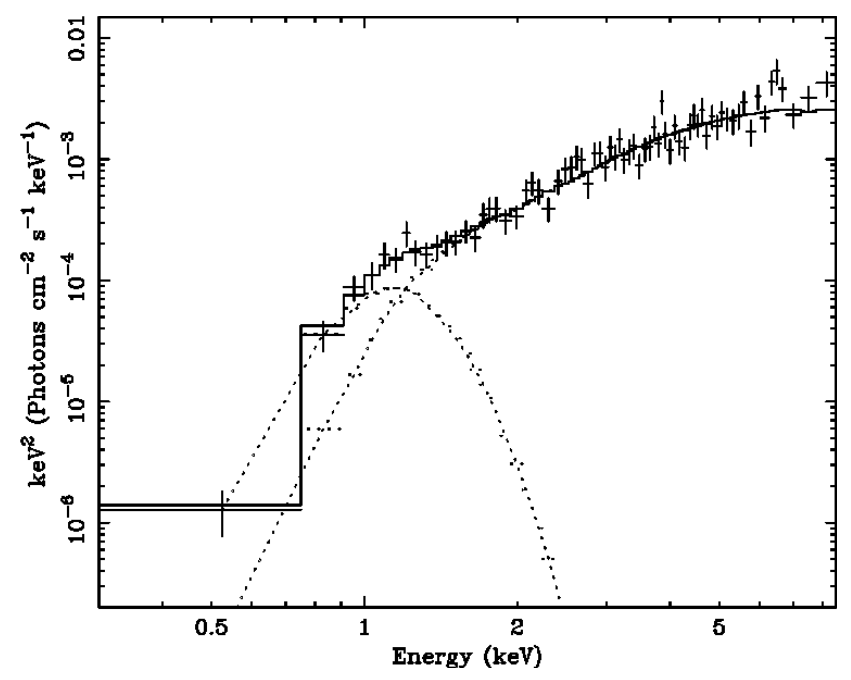

Fig. 4. Best-fit spectrum of the CV RX J2015.6+3711 (see details in the text).

the blazar at high energies. One solution is to assume that the BAT/IBIS emission is due to the combined contribution of two objects, i.e. the blazar, characterised by a simple power law and the $\mathrm{CV}$, described by a bremsstrahlung component. By doing this and letting the photon index of the blazar power law vary within the observed boundaries (1.44-1.88), it is possible to put more reasonable constraints on the $\mathrm{CV}$ bremsstrahlung temperature, which falls in the range $13-15 \mathrm{keV}$, i.e. closer to the values typically observed in this type of binary systems (Landi et al. 2009). This information can in turn be used to describe the $\mathrm{CV}$ average spectrum seen by XRT. Adopting the complex model described above (i.e. black body plus partially absorbed bremsstrahlung both passing through the Galactic column density), a good fit $\left(\chi^{2} / v=70.5 / 65\right)$ is obtained with a black body temperature of $\left(0.121_{-0.20}^{+0.29}\right) \mathrm{keV}$ and a column density of $\left(8.8_{-4.1}^{+6.1}\right) \times 10^{22} \mathrm{~cm}^{-2}$ covering $\left(0.64_{-0.06}^{+0.07}\right) \%$ of the source (see Fig. 4); the 2-10 keV flux is $\sim 4.4 \times 10^{-12} \mathrm{erg} \mathrm{cm}^{-2} \mathrm{~s}^{-1}$, while its extrapolation to high energies gives a $20-100 \mathrm{keV}$ flux of $\sim(2.1-2.6) \times 10^{-12} \mathrm{erg} \mathrm{cm}^{-2} \mathrm{~s}^{-1}$. A closer inspection of Fig. 4 indicates the presence of excess emission around $6.4 \mathrm{keV}$. The addition of an iron line is required by the data at $99 \%$ c.l. and provides an energy centroid $E=\left(6.48_{-0.08}^{+0.10}\right) \mathrm{keV}$ and an equivalent width $E W=\left(480_{-246}^{+210}\right) \mathrm{eV}$.

The overall picture which therefore emerges from the analysis of the XRT spectral data leads one to conclude that IGR J20159+3713/SWIFT J2015.9+3715 is likely the unresolved emission of two nearby high-energy emitters, the hard X-ray blazar B2013+370 and the likely magnetic CV RX J2015.6+3711. Purely on the basis of spectral information and data extrapolation, we estimate that the former contributes around $80-90 \%$ of the hard X-ray flux, while the latter provides the remaining $10-20 \%$ of $20-100 \mathrm{keV}$ photons.

For the sake of completeness, we have also performed the spectral analysis of the X-ray emission associated with CTB 87, which has recently been studied in details by Matheson et al. (2013) exploiting Chandra observations. Also in this case all XRT data have been added together to study the average X-ray spectrum of this source. Unfortunately, due to the lower XRT spatial resolution, we cannot perform a space-resolved analysis with the same accuracy of Chandra, but have nevertheless been able to study the spectral properties of the entire system as well as of the central compact region. The spectrum of the entire object is well fitted with a simple power law absorbed 
by Galactic absorption and is characterised by the following parameters: a photon index $\Gamma=(1.78 \pm 0.15)$ and an observed $2-10 \mathrm{keV}$ flux of $\sim 2 \times 10^{-12} \mathrm{erg} \mathrm{cm}^{-2} \mathrm{~s}^{-1}$. This is fully compatible, within uncertainties, with the results found by Matheson et al. (2013) using Chandra data (see total diffuse emission in their Table 2). Restricting the analysis to a region of 20 arcsec around the brightest XRT pixel, we obtain instead a slightly flatter spectrum $(\Gamma=1.42 \pm 0.22)$ and a lower $2-10 \mathrm{keV}$ flux $\left(\sim 8 \times 10^{-13} \mathrm{erg} \mathrm{cm}^{-2} \mathrm{~s}^{-1}\right)$. Our central region includes the putative pulsar, the compact nebula, and the inner diffuse emission region resolved by Chandra (Matheson et al. 2013); also in this case our spectral parameters compare well with those of Chandra despite the lack of the same resolution in our data. In particular, we confirm the flattening of the X-ray spectrum when only the central part of CTB 87 is considered. As a final remark, we note that Matheson et al. (2013) used two Chandra observations which they merged together to enhance the signal to noise ratio, thus missing information on variability. Given enough statistics, it would be interesting to explore which of the various features detected by Chandra in the source inner region is responsible for the flux variations seen by XRT.

\section{Connection to the GEV/TeV emission}

Starting from the above discussion, it is possible to move forward and assess the relation between IGR J20159+3713/SWIFT J2015.9+3715 and the GeV/TeV emitters. The IBIS/BAT emission, being mostly due to the blazar, is clearly connected to the GeV object. The SED of B2013+370 is shown and discuss by Kara et al. (2012): data above $20 \mathrm{keV}$ are potentially interesting as they can provide a way to discriminate between different emission scenarios. Using the blazar contribution estimated in the present paper, we favour a scenario where the source is powered by a leptonic model with the synchrotron self Compton (SSC) component dominating at X-rays and the external Compton (EC) one providing the gamma output. More specifically, the data above $20 \mathrm{keV}$ are significantly below the SSC+EC2 model favoured by Kara et al. (2012) (i.e. that with a lower temperature external field), and are instead more compatible with the SSC+EC1 model (i.e. that with a higher temperature field). We also note that the IBIS/BAT spectrum is relatively flat and more compatible with that expected in the case of the SSS+EC1 model. Clearly, variability and source contamination is an issue in this case and for a more precise analysis of the source SED not only simultaneous data are necessary, but also better angular resolution at the highest X-ray energies. Nevertheless, it is interesting to underline here the importance of hard X-ray information for such type of studies.

As for the CV contribution to the Fermi source, this is very likely negligible, if not null, although one must remember the case of XSS J12270-4859. This object has also been detected at hard X-ray energies by INTEGRAL/IBIS and Swift/BAT, it has then be classified as a CV of the magnetic type (Masetti et al. 2006) and subsequently it was associated with a Fermi source (1FGL J1227.9-4852/2FGL J1227.7-4853) listed in the first and second catalogues (Abdo et al. 2010, 2012); only recently, it was described as a peculiar type of X-ray binary (De Martino et al. 2010, 2013; Hill et al. 2011). Furthermore, XSS J12270-4859 is not the first time that a CV has been associated with a GeV emitter. PSR J1023+0038 is another GeV emitter initially believed to be the first radio-selected CV (Thorstensen \& Armstrong 2005; Bond et al. 2002) and only after closer scrutiny classified as a pulsar, in much the same way as XSS J12270-4859 was initially described as a CV and then found to be a peculiar object. This is simply to caution the reader that although RX J2015.6+3711 is an unlikely contributor to the Fermi source, it should be studied more in depth before it can be totally disregarded as a $\mathrm{GeV}$ emitter. This is even more true in view of its X-ray flux variability, which is clearly present in the XRT data. The scenario at TeV energies is by far simplier as the VERITAS TeV source is most likely associated with CTB 87 and related either to the SNR, its associated pulsar/PWN or both. In this case, no association is implied with the INTEGRAL/Swift high-energy object.

\section{Conclusions}

In this paper we have discussed the nature of the newly discovered INTEGRAL/Swift source IGR J20159+3713/SWIFT $\mathrm{J} 2015.9+3715$ which lies in a complex region of $\mathrm{GeV} / \mathrm{TeV}$ emission.

Through X-ray follow-up observations with Swift/XRT, we have been able to identify this new detection with the combined emission of the blazar B2013+370 and the CV RX J2015.6+3711. Both objects are variable in X-rays, with the $\mathrm{CV}$ being the most variable of the two. The emission above $20 \mathrm{keV}$ is likely dominated by $\mathrm{B} 2013+370$, but the contribution from RX J2015.6+3711 is not small. The blazar emits up to $\mathrm{GeV}$ frequencies where it is seen by Fermi, while the CV temperature is too low to provide any contribution at these high energies. Finally, the INTEGRAL/Swift source is not associated with the $\mathrm{TeV}$ emission which is most likely due to the SNR/PWN CTB 87.

Acknowledgements. We acknowledge finacial support from ASI under contract ASI I/033/10/0 and ASI/INAF I/009/10/0. This research has made use of the NASA/IPAC Extragalactic Database (NED) operated by Jet Propulsion Laboratory (California Institute of Technology) and of the HEASARC archive provided by NASA's Goddard Space Flight Center. The authors also acknowledge the use of public data from the Swift data archive.

\section{References}

Abdo, A. A., Ackermann, M., Ajello, M., et al. 2010, ApJS, 188, 405 Abdo, A. A., Ackermann, M., Ajello, M. et al. 2012, ApJS, 199, 31 Aliu, E. (for the VERITAS Collaboration) 2011, Proc. 32nd ICRC, Beijing, China [arXiv: 1110.4656$]$

Baumgartner, W. H., Tueller, J., Markwardt, C. B., et al. 2013, ApJS, 207, 19 Bond, H. E., White, R. L., Becker, R. H., \& O'Brien, M. S. 2002, PASP, 114, 1359

Burrows D. N., Hill, J. E., Nousek, J. A., et al. 2005, Space Sci. Rev., 120, 165 Cusumano, G., La Parola, V., Segreto, A., et al. 2010, A\&A, 510, A48 De Martino, D., Falanga, M., Bonnet-Bidaud, J.-M., et al. 2010, A\&A, 515, A25 De Martino, D., Belloni, T., Falanga, M., et al. 2013, A\&A, 550, A89 Halpern, J. P., Eracleous, M., Mukherjee, R., \& Gotthelf, E. V. 2001, ApJ, 551, 1016

Hartman, R. C., Bertsch, D. L., Bloom, S. D., et al. 1999, ApJS, 123,79

Hill, J. E., Burrows, D. N., Nousek, J. A., et al. 2004, Proc. SPIE, 5165, 217

Hill, A. B., Szostek, A., Corbel, S., et al. 2011, MNRAS, 415, 235

Kalberla, P. M. W., Burton, W. B., Hartmann, D., et al. 2005, A\&A, 440, 775

Kara, E., Errando, M., Max-Moerbeck, W., et al. 2012, ApJ, 746, 159

Krivonos, R., Tsygankov, S., Revnivtsev, M., et al. 2010, A\&A, 523, A61

Krivonos, R., Tsygankov, S., Lutovinov, A., et al. 2012, A\&A, 545, A27

Landi, R., Bassani, L., Dean, A. J., et al. 2009, MNRAS, 392, 630

Masetti, N., Morelli, L., Palazzi, E., et al. 2006, A\&A, 459, 21

Matheson, H., Safi-Harb, S., \& Kothes, R. 2013, ApJ, 774, 33

Moretti, A., Campana, S., Tagliaferri, G., et al. 2004, Proc. SPIE, 5165, 232

Page, K. L., Reeves, J. N., O'Brien, P. T., \& Turner, M. J. L. 2005, MNRAS, 364, 195

Ptak, A., \& Griffiths, R. 2003, Astronomical Data Analysis Software and Systems XII, eds. H. E. Payne, R. I. Jedrzejewski, \& R. N. Hook, ASP Conf. Ser., 295, 465

Safi-Harb, S. 2012, Proc. of the 5th International Meeting on High Energy Gamma-Ray Astronomy, AIP Conf. Proc., 1505, 13

Tavecchio, F., Maraschi, L., Ghisellini, G., \& Kataoka, J. 2007, ApJ, 665, 980

Thorstensen, J. R., \& Armstrong, E. 2005, AJ, 130, 759 